\title{
Le scénario français en quête d'auteurs (1908-1918)
}

French screenwriting in search of authors

\section{Alain Carou}

\section{OpenEdition}

\section{Journals}

Édition électronique

URL : https://journals.openedition.org/1895/4432

DOI : $10.4000 / 1895.4432$

ISSN : $1960-6176$

\section{Éditeur}

Association française de recherche sur l'histoire du cinéma (AFRHC)

\section{Édition imprimée}

Date de publication : 1 décembre 2011

Pagination : 28-51

ISBN : 978-2-2913758-67-4

ISSN : 0769-0959

\section{Référence électronique}

Alain Carou, «Le scénario français en quête d'auteurs (1908-1918)», 1895. Mille huit cent quatre-vingtquinze [En ligne], 65 | 2011, mis en ligne le 01 décembre 2014, consulté le 15 avril 2022. URL : http:// journals.openedition.org/1895/4432 ; DOI : https://doi.org/10.4000/1895.4432

\section{(c) $\mathrm{AFRHC}$}




\section{ÉTUDES}

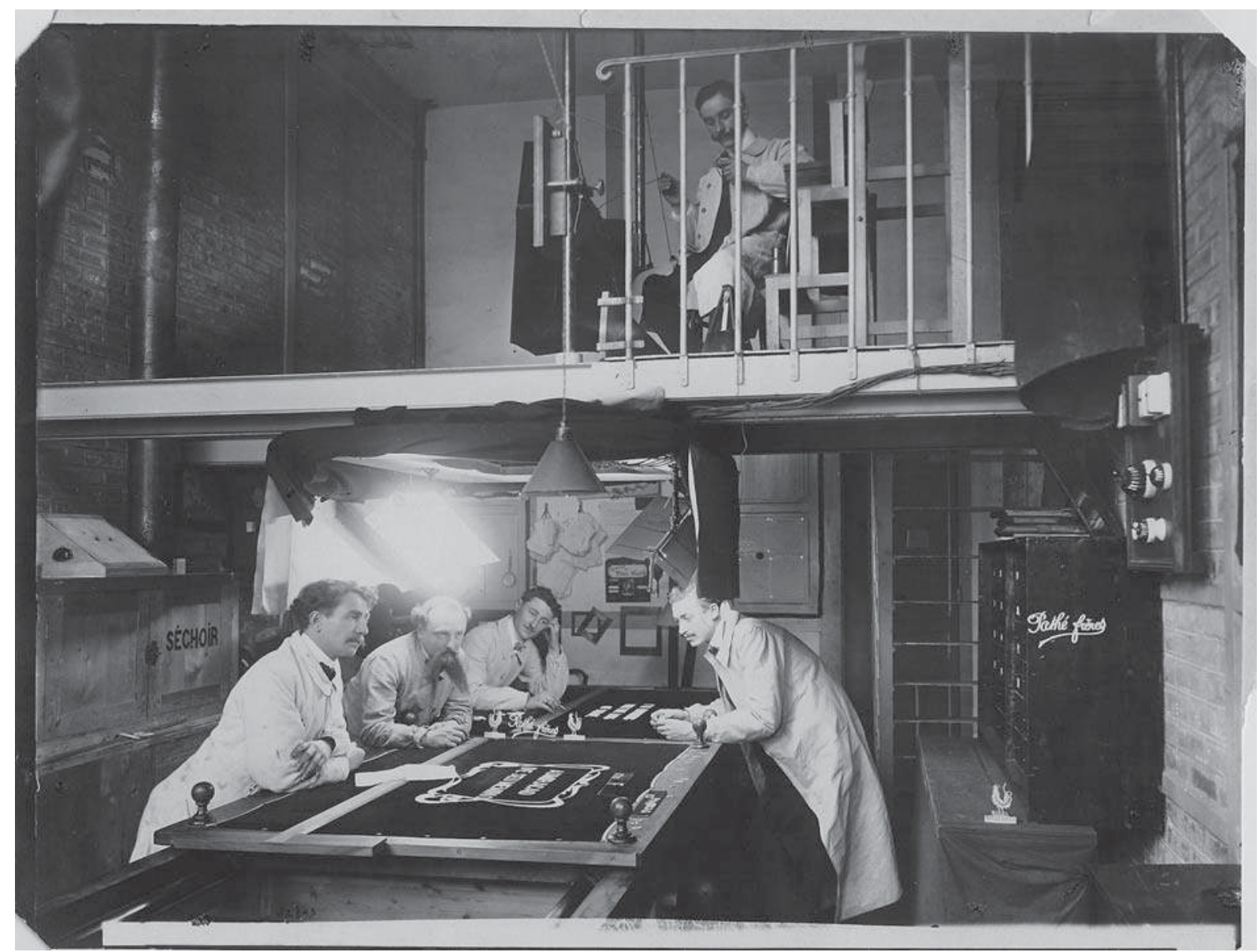

Filmage des titres chez Pathé. Photographe : J. David. Coll. Fondation Jérôme Seydoux-Pathé. 


\title{
Le scénario français en quête d'auteurs ( 1908-1918)
}

\author{
Par Alain Carou
}

En 1941, l'écrivain Jean Joseph-Renaud confie au magazine Vedettes ses souvenirs du cinéma d'avant 1914. Le tableau qu'il brosse de la maison Pathé, à laquelle il essayait de vendre des scénarios ${ }^{1}$, ne manque pas de saveur: le comité de lecture se composait «d'un ancien boucher, d'un vieux cabot de banlieue et d'une manucure retirée», et les scénarios amenés par les jeunes écrivains étaient lus à haute voix afin qu'une dactylographe dissimulée derrière un paravent les prît en note en cachette ${ }^{2} .$. Quoique le trait soit sans doute forcé, le texte témoigne du choc des cultures que fut la rencontre des milieux littéraires avec les éditeurs de films.

Cependant, les origines sociales et les manières de se conduire ne suffisent pas à expliquer les tensions qui caractérisent leurs relations pendant la seconde décennie du cinéma. Plus fondamentalement, l'absence de définition d'un scénario reconnue par tous a entretenu au moins deux types de malentendu ou de désaccord. Le premier concerne la qualité principale attendue d'un scénario: devait-il avant tout proposer une idée originale (en termes d'histoire ou d'effets) ou bien fournir un plan de tournage fonctionnel prêt à l'usage? Au sein même de l'industrie cinématographique, la réponse a pu varier au fil du temps et selon les acteurs (éditeurs de films ou presse corporative, notamment). De là découle logiquement la préférence pour un recrutement ouvert à quiconque est susceptible d'avoir l'idée d'une bonne histoire, ou réservé à des professionnels bien au fait des règles d'écriture. Cette question n'a jamais été résolue de manière univoque, jusqu'à aujourd'hui. Mais tant que ces règles ont relevé d'un savoir-faire non formalisé, et même lorsqu'elles ont tout juste commencé à être formulées et ne constituaient donc pas encore un socle commun, tout le monde est loin d'avoir eu même clairement conscience de ces options divergentes.

Le second désaccord concerne la rémunération. Comment évaluer cette production de l'esprit, inscrite sur quelques feuillets, qu'est un scénario? Cette question pourrait paraître très liée à la précédente. Ce n'est pourtant pas tellement le cas, tant ont primé les raisonnements par analogie avec d'autres modes de rémunération comme le théâtre et l'édition ou la tarification du travail à la pièce. Il en résulte des tensions et des regroupements d'intérêts qui participent de la constitution d'un groupe professionnel, tout comme le processus de spécialisation technique, mais suivant des logiques et des lignes de partage qui ne sont pas nécessairement les mêmes.

1. Rappelons que le terme de "scénario" a été emprunté au théâtre où il était d'usage courant. Arthur Pougin le définit en 1821 comme "plan divisé acte par acte, scène par scène, tel qu’enfin il doit être exécuté, mais ne donnant qu’un résumé et un récit de l'action dramatique, et non point le dialogue des personnages qui prennent part à cette action" ( cité par Jean Giraud, le Lexique français du cinéma, des origines à 1930, Paris, CNRS, 1958, p. 178).

2. Jean Joseph-Renaud, Vedettes, n 8,8 mars 1941. On aura reconnu en l'ancien boucher Charles Pathé et dans le cabot Ferdinand Zecca. (Merci à Stéphanie Salmon pour m’avoir signalé cet article). 


\section{Sous-traitance contre scénario d'auteur}

La division du travail entre la conception des sujets de films et leur mise en scène est liée au développement et à l'industrialisation de la production. L'entrée d'André Heuzé chez Pathé en 1904 en est le premier signe connu ${ }^{3}$, mais par la suite on dispose de très peu d'informations sur les salariés des éditeurs de films chargés d'écrire des scénarios. Il est en tout cas certain que les éditeurs se reposent de manière croissante sur la sous-traitance.

On peut définir comme sous-traitance le fait, pour une entreprise, de déléguer une partie du processus de production à un tiers qui dispose de compétences qu'elle n’a pas. Le sous-traitant doit respecter les normes de production définies par le commanditaire. Son travail est payé à forfait. Sa contribution est anonyme: le produit fini porte uniquement la marque de fabrique du commanditaire.

On dispose aujourd'hui d'un ensemble important de scénarios des années 1907-1909, quand plusieurs firmes ont commencé à les déposer régulièrement auprès de la Bibliothèque nationale, sous forme dactylographiée, pour tenter de se protéger de la contrefaçon ${ }^{4}$. Ces documents témoignent-ils de l'état original des scénarios livrés à ces firmes, ou ont-ils été réécrits entre-temps par les services en charge de la mise en scène? L'objectif même de ce dépôt incite à pencher pour la première hypothèse: plus le dépôt sera effectué rapidement, moins seront grandes les chances de voir une idée pillée ou vendue une seconde fois à un concurrent par le même fournisseur. L'examen des scénarios Pathé des années 1907-1909 semble corroborer cette hypothèse. L'hétérogénéité de la mise en page des scénarios dactylographiés révèle non seulement qu'il n'existe pas de bureau spécialisé dans cette tâche ${ }^{5}$, mais que les dactylographes ont saisi des documents manuscrits eux-mêmes présentés de manières multiples ${ }^{6}$. Il arrive aussi, par une exception instructive, qu'un scénario ait fait l'objet d'un double dépôt. Tel est le cas de Rêve du joueur ${ }^{7}$. Il est probable que le scénario a d'abord été déposé juste après avoir été acquis auprès d'un fournisseur peu habitué à la description des trucs, et l'a été à nouveau une fois qu'il a été relu et sa rédaction clarifiée par le service de la mise en scène. Preuve que le dépôt était effectué rapidement, au risque de devoir y revenir.

3. On notera d'ailleurs que quasiment au même moment, en 1903, un autre jeune écrivain, Georges Fagot, est engagé comme secrétaire de Zecca et se trouve alors en charge des sous-titres à la place de l'ancien opérateur Caussade (Francis Ambrière, «À l'aube du cinéma. Les souvenirs de Georges Fagot», l'Image, n 30, 5 août 1932).

4. Sont attestés quelques dépôts plus anciens de Charles Urban, en 1905. On distingue deux types de textes envoyés par Pathé frères au dépôt légal des estampes. Les résumés «conformes à la vue» sont déposés en lieu et place du film fini, la B.N. n'étant pas à même de recevoir le dépôt de bobines. Accompagnés le plus souvent (mais pas toujours) de bandes de photogrammes, ils peuvent, dans les premières années, prendre la forme d'un véritable découpage analytique d'après visionnement, même si les synopsis commerciaux s’imposent par la suite. Les scénarios avant tournage sont déposés, eux, en contradiction avec l'esprit du dépôt légal, qui s'applique uniquement aux publications - les éditeurs de films profitant sans doute d'un manque de compétence et d'intérêt de la B.N. pour être à même d'opérer la distinction.

5. Laurent Le Forestier, Aux sources de l'industrie du cinéma. Le modèle Pathé, 1905-1908, Paris, AFRHC/L'Harmat$\tan , 2006$, p. 244.

6. On ne s'expliquerait pas, par exemple, des numérotations de tableau en retrait de marge - opération nécessitant une manipulation supplémentaire pour la dactylographe - si le document reproduit n'était pas présenté aussi de cette manière. 7. Rêve du joueur (1908), BnF, Arts du spectacle, $4^{\circ}-\mathrm{COL}-4$ (650). Laurent Le Forestier cite d'autres exemples (op. cit., p. 269). 


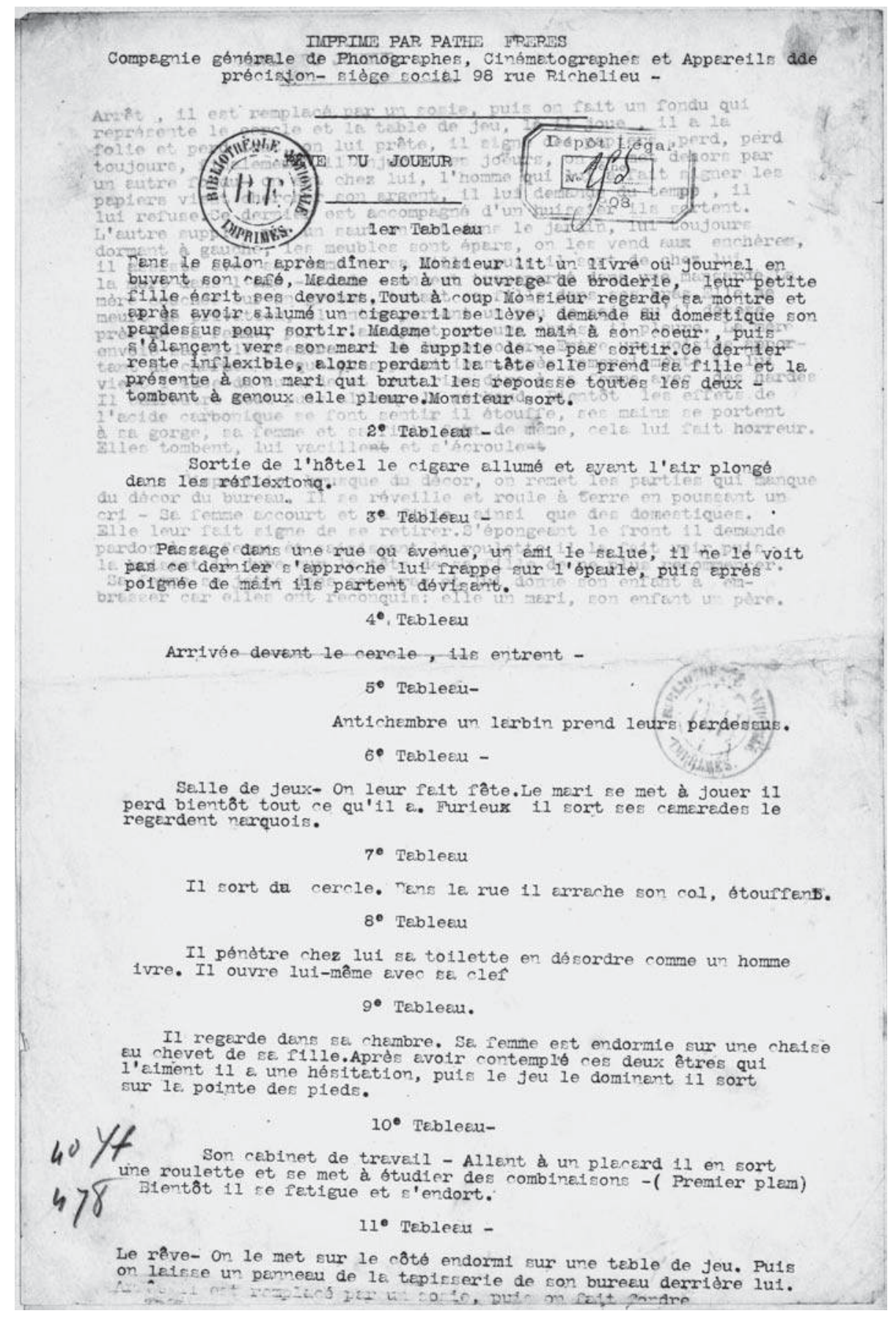

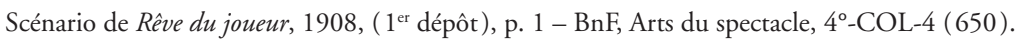


REVE TU JOUEUR.

Arrêt, 11 est remplacé par un soste, puis on fait un fondu qui représente le cercle et la table de jeu, là 11 joue, 11 a la perd folle et perd tout, on lul prete, les jofurs, on le met dehors par thome qui lui a fait sismer les un eutre fondu- on est chez argent, 11 lui demende du tempp, il papiers vient chercher son argent, 11 lui demende du tempp, 11 Iui refuse. Ce dermier est accompagné d'un huissier ils sortent. L'autre supplie par un seut on est dans le jerdin, lui toujours 11 assiste avec sa femme et sa fille, puis 11 sort de chez lui la mort dans l'ane - fondu - on est transporté dans une mansarde. La mère couchée sur un grabat, tenant sa fille dans ses bras.slle se meurt de privation, 11 entre portant un peu de pain qu'il dépose près de sa femme, puis s'asseyent sur une cheise 11 pleure. La mère envole sa fille $1^{\prime}$ embresser et le consoler. Entre une voisine appor tant du cherbon et quelques provisions.Après remerciements il lui vient l'idée du sulcide et sans rien dire à sa femme avec des hardes Il calfeutre tout puis allume le réchaud. Bientôt les effets de I'acide carbonique se font sentir il étouffe, ses mains se portent

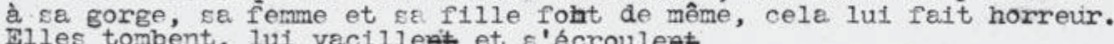

Nisparition brusque du décor, on remet les parties qui manque du décor du bureau. Il se réveille et roule à terre en poussant un cri - Sa femme accourt et sa fille ainsi que des domestiques. vile leur fait signe de se retirer. S'epongeant le front 11 demande pardon à sa femme et saisissant la roulette 11 la fait voir puis la brisant 11 jure sur la tête de sa fille de ne plus recommencer. Sa femme se jette dans ses bras et lui donne son enfant à embrasser cer elles ont reconquis: elle un meri, son enfant un père.

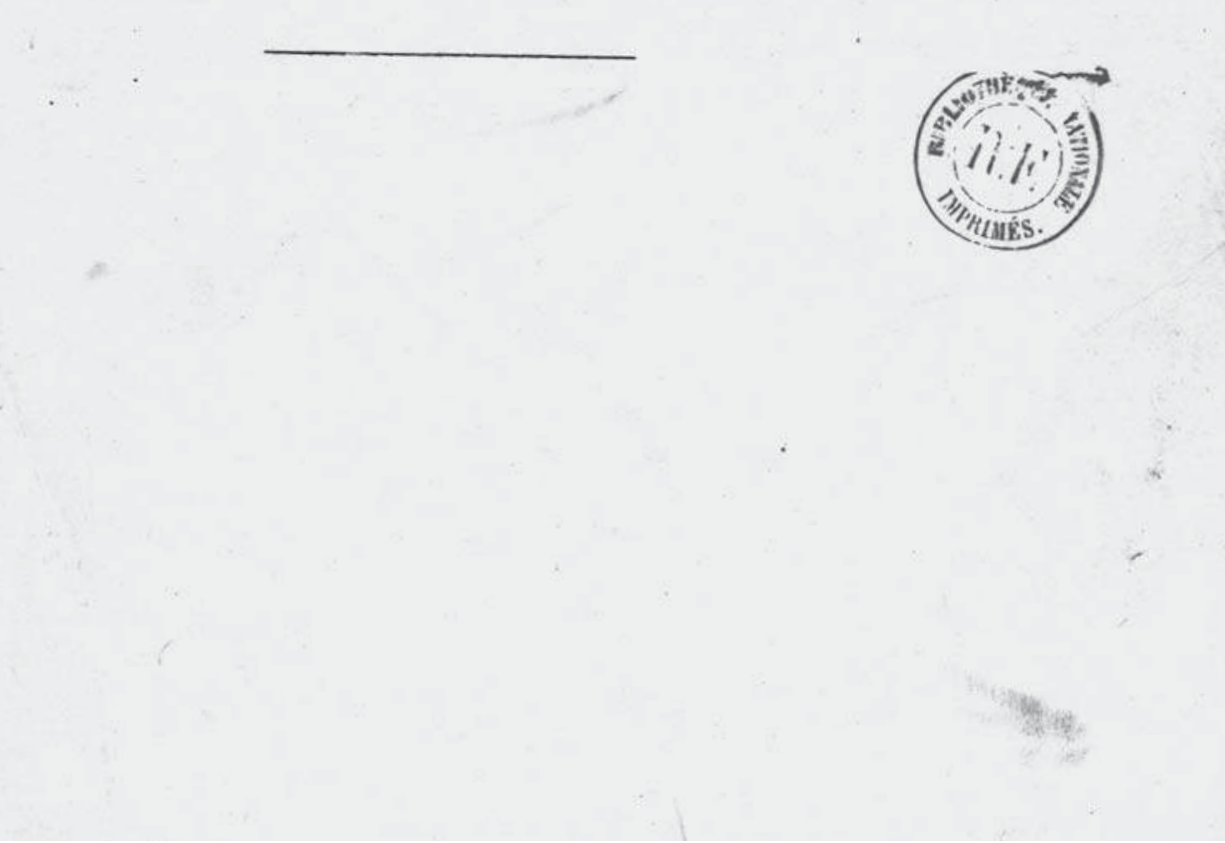

Scénario de Rêve du joueur, 1908, ( $1^{\text {er }}$ dépôt), p. 2 - BnF, Arts du spectacle, $4^{\circ}$-COL-4 (650) 
TIMPIIT PAR PATHE FRBRTSS

Compagnie Générale de Phonographes, Oinematographes et Appareils de Préclsion.- Siège Social: 98, Rue de Richelieu PARIS.

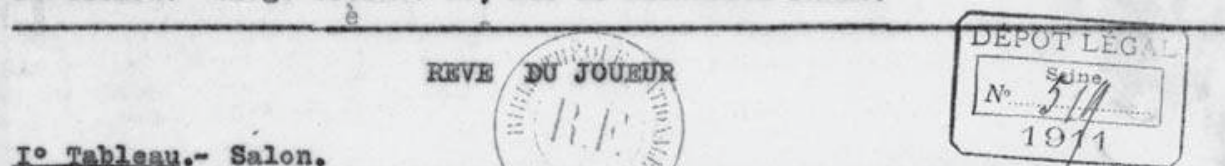

Après dîner, Pierrot 11t une lettre ou un journal, Ladame ost à un travaì de broderie; leur petito fille éerit ses devoirs. Tout à coup Uonsieur regaxde sa montre et après avo1r allumé un oigare 11 se lève, demandant au domestique son pardessus pour soriir.- Hadame Pierrot serabie malheureuse de voir que Pierrot va encore abandonner la maison et $s^{\prime}$ avançant vers lui, elle le supplie de ne pas sortir. Ce dernier reste inflexible, alors pexdant la tête, elle prend sa fllle et la présente à son maxi qui, brutal, les repgusse toutes deux, puis tombant aे genoux, l'enfant sur son épaule, elles pleurent toutes deux. Pierxot sort.

20 Tablealle- Soattie de i'hôtel.

Pierrot allume un gigare, après avoix réfléch1\%, 11 fait un geste désinvolte et part.

30 Tableau.- Une salle de jeu.

On Pait fête et on I'invite à $s^{*}$ asseoir; 11 se met à jouer: intercaler un Io plan.- Il perd tout ce qu'il a.- Revenir au déoor. Furieux 11 se lève et sort.

40. Tableau.- Sortie du cercle, dans 1a rue.

Il arrache son col, étouffant.

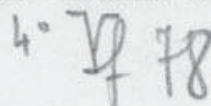

50 Tableau.- Ia chambre de son enfant. -A drolte, un secrétaire en évidence Ba fomne s'est endormíe sux une chaise, au ohevet de sa pille. Après avoir comtemplé ces deux ếtres qu'il aime il a une hésitation, puis 10 jeu dominant, 11 past sux la pointe des pieds dans une autre pièce.

60 Tableau- Cabinat de travail de Pierrot.

Allant a un placard 11 en sort une roulette, la place sur la table et se met à étudier des combinaisons. I० Plan Il se met à jouer mais blentôt il se fatigue ot s'endort.

Lorsqu'1l $\mathrm{y}$ a deux ou trois mètres qu'11 est endorm1, remplacer Plerrot endorni sur la table Io plan (faire un fondu) par un figurant habilié comane lui et s'asseyant spux le figurant, 11 se lève lentement dès que commence le second fondu pour permettre a. Pierrot de se dédoubler 'est à dire que le publio le voit se levex en même temps qu'en réalité 11 reste endorni sux sa roulette; debout, à côté de la table, 11 semble rérléchir, prend une vague détermination.et sort sur la pointe des pieds.

70 Tableak, La chambre de sa P11le.-

Is mère est toujours endormie. Arxive Plexrot qui se dirige vers le secrétaire, puis $I^{\circ}$ ouvrant 11 vole tout l'argent disponible et se dirige vers la porte de sortie.

70 Tableau.- Salle de jeu, la table de jeu.

Assez premier plan. Une place inocoupée, les gens jouent. In fondu Pieræot apparaft à la place vide, ceoi pour rappeler au public que nous sommes en plein rêve. Plerrot joue et perd; en face de lui un individu 
à 2a physionomie bien stylisée gagne tout ce que Plertot perd. Lorsqu'11 a perdu, Pierrot se lève sombre * 11 réf léchit à la mauvalse action qu' $12 \perp$ vient de comettre.- L' Individu qui a gagne empoche, se lève et sort. pierrot regarde dans sa direotion et a poux lui un mauvais regard et il le suit.

80 Tableau.- I'antichambre du cercle.

i individu prend son pardessus et sort. Plerrot arrive, prend son manteau ot serablo se diriger dans $l_{\mathrm{a}}$ direotion du dernier.

90.Tableau.- Une rue.

Nous voyons arriver I'individu, suivi de Plerrot qui se dissimule le long d'un mux. Arrivé en premier plan, Pietrot se jotte sur lus et pris $\mathrm{d}^{\prime}$ une rage Poile, il l'étrangle, le dévalise et part vers le lointain, non sans se retourner de oxainte d'être poursuivi.

I0 Trableau.- Ia salle de jeus

Pierrot revient prendre place et se remet à jouer mais ì ce moment doux homes d'arraes et un comisalsaire arrivent, examinent toug les assistants et le commissaire désigne de sa canne Pierrot, Ies deux homises d'aries $I^{\prime}$ arrêtent et $I^{\prime}$ 'emmènent, malgré ses protestations.

110. Thbleav.- Une prison;

Les hommes d'armes emmènent pierrot, le jettent dans la prison et partent. Celui-ci s'assied, examine les murs ot semble dire: nYo1 où me mène ma mauvaise conduite", puis pleure dans ses mains.- Premier plan de mêne dimension que 1e premier plan du Plerrot dे lo roulette, 11 devra être assis de Paçon ̀̀ être de même grosseux et à la mêne place sur I'éoran.- Pierrot après avoix pleuré, relève la tête puis repasso on sa mémoire toutes les mauvaises actions qu'11 vient de comiettre. IIjmine la soène du vol, 11 voit sa femue tombant de fatigue au chevet de son enfant, le jeu où il perd, le orine, l'homine étendu dans la rue, $\lambda^{\prime}$ arrestation puis se voit juger et pendu. - Au moment où mime qu'on va Iui mettre la corde au cou et qu'il va être pendu, Pierrot a un geste de désespoir et cachant son visage dans ses mains, il sanglotte, en baisant la tête. Arrêt.- Nous revenons au prenier plan, Pierrot est, endorai sur sa roulette, c'est la réalité. Dans un mouvement d'ef froi il s'est éveillé ot se xetrouve couché sux la table (xevenix au décor dans son entier) il rérléchity pense au mauvais rôve qu'12 vient do $r a i x e$, se deanande si ce n'est pas la réalité, puis se rendent compte que sa triste pession pour lo jeu peut être cause de pareils malheurs $11 \mathrm{~s}$ 'empare de la roulette t $1 \mathrm{a}$ brise. Quelques instants, après, Ea femne qui a entendu du bruit par ait, semble étonnée, Plerrot $s$ 'empare des débris de sa roulette, les lui montre, puis I'entralne dans la chambro de con enfant; posant les débris de sa roulette à terre 11 met les pieds dessus at fait jo mexment sur la tête de sa petite Plile qu'il ne jouera jamais plus. Sa femtre se jette dans ses bras, puis il embrasge son enfant. Miles ont reconquis, elle un maxi et la petite Plile un père. 
Puisqu'il semble donc juste de supposer que les scénarios étaient déposés à la B.N. sans avoir été réécrits, ils attestent qu'en 1907 déjà, les fournisseurs indépendants de Pathé, ou du moins les plus réguliers, ont intégré les éléments d'un "cahier des charges»: découpage en tableaux, valeurs de plans, personnages et situations génériques ${ }^{8} .$. À côté de ces premières normes d'écriture, on glisse facilement d'un descriptif des tableaux, enrichi de propositions de mise en scène, à un argument relatif à la faisabilité du film, par exemple la manière de se procurer les accessoires ou le fait que le sujet se prête à un tournage en n'importe quelle saison. L'un des plus anciens textes sur la question du scénario parus dans la presse corporative présente comme deux phases distinctes l'examen par l'éditeur des idées que lui présentent tous les jours des "auteurs spéciaux", puis, une fois l'idée acceptée, l'examen des difficultés d'exécution: «si le film peut se faire dans la saison, quels sont les déplacements nécessaires, quelle sera la dépense pour les décors, costumes et accessoires, combien d'artistes seront nécessaires »" En réalité, la question de la facilité d'exécution est déjà en jeu dès la négociation entre le fournisseur et l'éditeur.

En régime de sous-traitance, la fourniture d'éléments d'évaluation technique joue aussi un rôle dans la négociation financière. Que vaut le scénario? L'indication d'un métrage estimatif tableau par tableau ${ }^{10}$ indique certainement une évolution du cahier des charges des scénarios à un moment où Pathé frères cherche à maîtriser les coûts de production ${ }^{11}$. Mais si le fournisseur réussit à persuader l'éditeur que son scénario peut servir de base à un film long, son prix d'acquisition sera valorisé en proportion. En témoigne la relation satirique, par Armand Massard, d'une discussion «surprise» par lui dans le métro: un éditeur et un écrivain discutent le nombre de mètres que va produire un scénario et le prix d'achat qui en résulte ${ }^{12}$.

Les noms des fournisseurs de scénarios restent fréquemment inconnus du public. Ils ne sont mentionnés ni sur les affiches, ni sur les programmes, ni dans les génériques. Pourtant, ils apparaissent fréquemment sur les copies dactylographiées déposées à la B.N., à gauche du titre. On les trouve même ajoutés à la main sur la copie quand la dactylographe les a omis: il ne s'agit donc pas d'une indication anodine. À quoi bon garder trace du nom d'un «auteur" pour l'effacer ensuite, une fois le film fait? Le plus probable est que le dépôt légal ne soit qu'une des destinations (et même une destination secondaire) de la «multigraphie» des scénarios et que sa fonction principale soit de permettre de constituer au sein des maisons de production des bibliothèques de scénarios faciles à exploiter. Les noms des fournisseurs servent de critère d'indexation ou de classement de la bibliothèque de scénarios de Pathé. Se trouvent ainsi constitués des fonds de scénario "genre Gambart» ou "genre Rollini». Il se peut également que conserver trace du nom du fournisseur soit utile à l'éditeur pour lui permettre de se retourner contre lui en cas de plainte pour plagiat. On sait que la cession de scénarios s'accompagne de la signature par les fournisseurs d'« un papier pour [les] rendre responsables de toute cession similaire ou du plagiat $»^{13}$.

8. Laurent Le Forestier, op. cit., pp. 246-265.

9. Pierre de Jonckheere, "Pour faire un film», Kinema, n 5, 29 mars 1909.

10. Cf. par ex. Oh! les agents... les agents! (1908), BnF, Arts du spectacle, $4^{\circ}-\mathrm{COL}-4$ (366) : le métrage de chacun des 24 tableaux est fixé précisément.

11. Laurent Le Forestier, op. cit.

12. Armand Massard, "Propos d'âme simple», la Presse, n 6121, 16 mars 1909.

13. Raymond Laubier, "Contre les "flibustiers" du cinéma", le Courrier cinématographique, 3e année, n 14, 12 avril 1913. 


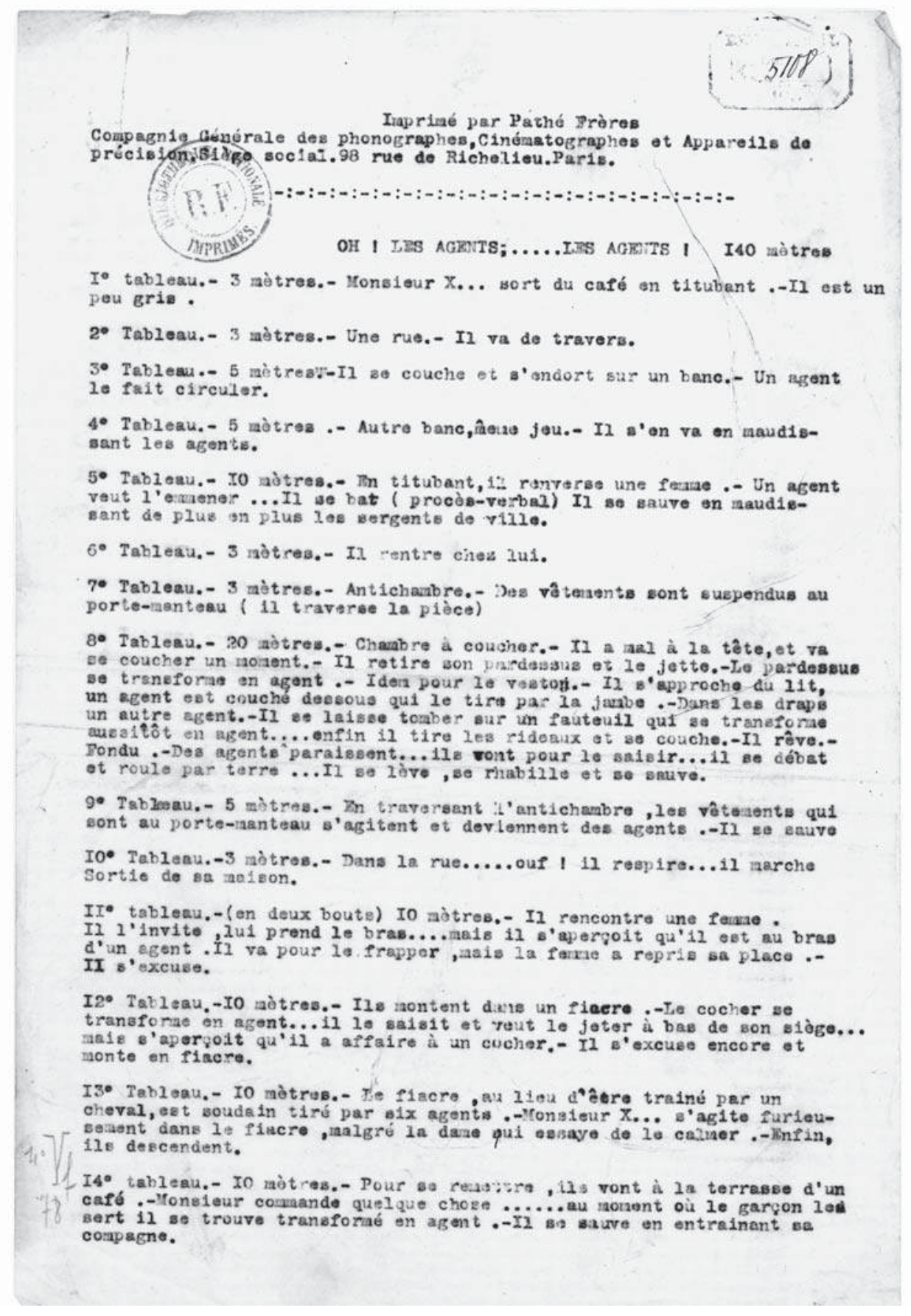

Exemple d'indication du métrage estimatif tableau par tableau - scénario de Ob! les agents... les agents!, 1908, $2 \mathrm{f}$ ( $1^{\text {er }}$ feuillet). BnF, Arts du spectacle, $4^{\circ}-$ COL-4 (366). 
En rupture avec la logique de sous-traitance, Le Film d'Art et la Société cinématographique des auteurs et gens de lettres (SCAGL), fondées en 1908, sont les premières à proposer des «scénarios d'auteur . Les signatures des rédacteurs de scénarios sont mises en valeur parce que, déjà renommées dans le domaine littéraire, elles peuvent servir à la promotion commerciale du film. En indexant leur rémunération sur le nombre de copies du film vendues ou louées ${ }^{14}$, Le Film d'Art et la SCAGL s'inspirent du système des droits d'auteur dans l'édition. En sollicitant très largement les milieux littéraires par l'intermédiaire de directeurs pleins d'entregent (Henri Lavedan, Pierre Decourcelle), les deux sociétés cherchent non à faire émerger un groupe de nouveaux spécialistes de l'écran, capables de rédiger un scénario conformément au cahier des charges de Pathé ${ }^{15}$, mais à recueillir des idées originales signées. Du même coup, par la moisson de contrats d'exclusivité qu'elles engrangent dès leur première année d'existence, ces deux sociétés s’arrogent aussi un quasi-monopole des films à «scénario d'auteur", fermant la porte à la concurrence. En quelques mois, la SCAGL s'arroge ainsi un monopole sur cent cinquante écrivains, qu'elle conforte en interdisant pratiquement à Pathé de se lier à toute société concurrente sur ce créneau ${ }^{16}$.

Dans la pratique, il n'est pas aisé d'assurer un flux régulier de scénarios prêts à tourner en s'approvisionnant auprès d'une pléiade d'écrivains connus et sollicités de manière occasionnelle. Le Film d'Art en fait l'amère expérience à ses débuts. Lavedan reçoit des propositions sophistiquées voire expérimentales, que Le Bargy, directeur de la scène, juge impossibles à mettre en scène, surtout avec des interprètes payés à prix d'or et peu disponibles. Elles sont donc finalement remisées après seulement quelques mois, au bénéfice de sujets simples et efficaces. Les scénarios en sont confectionnés, sous la direction d'Émile Fabre, par des écrivains débutants, payés à forfait et a priori non crédités ${ }^{17}$. Faute d'avoir su anticiper les écueils du scénario d'auteur, Le Film d'Art se tourne très vite en catimini vers une forme de sous-traitance.

\section{L'écriture de scénario, un travail profitable?}

De prime abord, l'écriture en sous-traitance représente un travail avantageux pour bien des auteurs dramatiques qui tentent de vivre de leur plume à Paris. Le prix moyen auquel est acheté un scénario se situe, selon plusieurs sources concordantes, autour de 50 francs $^{18}$. De 1907 à 1914, Henri de Brisay vend les siens à Louis Feuillade au tarif fixe de 50 francs pour les drames et 25 francs pour les

14. Ou plus exactement sur le métrage de film vendu, ce qui est une incitation à écrire des scénarios pour des films longs. 15. Les deux sociétés sont liées à Pathé qui édite toute leur production et à qui tous les scénarios sont donc soumis pour validation.

16. Le contrat entre les deux sociétés prévoit que Pathé ne pourra contracter avec une société produisant des films signés de membres des sociétés d'auteurs si cette société représente moins de quarante auteurs (contrats du 15 juin 1908 et du 3 décembre 1909, archives Fondation Jérôme Seydoux-Pathé).

17. Alain Carou, «Le Film d'Art ou la difficile invention d'une littérature pour l'écran (1908-1909)», 1895, n 56, «Le Film d'Art et les films d'art en Europe, 1908-1911» (Alain Carou et Béatrice de Pastre, dir.), décembre 2008, pp. 28-30. 18. José Germain, «Douze scénarios pour six cent francs», Pour vous, n 433, 4 mars 1937. Entretien avec Nora Jonuxi, Cinémathèque française, fonds de la Commission de recherches historiques, CRH-41. Rapport d'Adolphe Aderer sur la question du cinématographe, Annuaire de la Société des auteurs et compositeurs dramatiques, 1913-1914, p. 1050. 
sujets comiques ${ }^{19}$. En 1913, un fournisseur bien informé précise qu'un scénario se paie entre 20 et 100 francs, et exceptionnellement jusqu’à 500 francs $^{20}$. Un scénario rapporte ainsi à peu près autant que les droits d'auteur pour une représentation d'une pièce dans un petit théâtre parisien. Comme les firmes cinématographiques les achètent couramment par lots, le bénéfice apparait encore plus intéressant: José Germain trouve "pharamineux" les 600 francs versés d'un seul coup par Gaumont pour douze scénarios. La somme est en effet à comparer aux 500 francs de droits d'auteur par an - ou moins - dont doivent se contenter la plupart des jeunes auteurs dramatiques. Louis $Z$. Rollini, l'un des principaux fournisseurs de Pathé, perçoit même des «bonifications» sur les bandes qui se sont le mieux vendues, à l'instar de metteurs en scène et d'acteurs ${ }^{21}$.

Les auteurs dramatiques forment un tout petit monde, qu'on peut rencontrer dans les brasseries des boulevards, au voisinage des grands théâtres. Selon un témoignage tardif de Jean Joseph-Renaud, certains metteurs en scène se postent dans un café de la rue Auber et attendent qu'on vienne leur faire des propositions ${ }^{22}$. Des écrivains viennent leur présenter oralement leur idée de scénario. Si elle est acceptée, ils entreprennent alors de la développer par écrit. Quoiqu'enrobés de pittoresque, ces souvenirs cadrent bien avec ce qu'on peut reconstituer de la manière de travailler du jeune Abel Gance d'après les notes portées sur ses scénarios. Il destine d'abord l'idée du Glas du père Césaire à la SCAGL, au Film d'Art et à Éclair, avant de la proposer avec succès à Éclipse. Il a l'idée d'un film pacifiste mais veut «en parler [à un éditeur] avant de faire le scénario ". Il envisage de transformer un sujet dramatique, s'il est refusé par Camille de Morlhon, en scénario comique pour Max Linder. Les idées sont ainsi placées avant les scénarios développés, et un épisode abandonné à l'écriture devient à son tour une nouvelle idée à placer ${ }^{23}$. Dans l'Annuaire de la projection de Mendel (1914), on ne trouve à l'entrée «Auteurs de scénarios" que cinq noms obscurs, inconnus des filmographies existantes. Les éditeurs n'ouvrent pas un annuaire pour trouver des fournisseurs de scénarios: on traite de visu et entre personnes de connaissance. Lapparente facilité de la tâche et son caractère assez rémunérateur amènent de plus en plus de propositions de scénarios aux éditeurs. Seuls de rares nouveaux venus dans la production de films font parâ̂tre des annonces encourageantes, tel Harry: «Auteurs! Rappelez-vous bien que nous payons très cher les scénarios drames modernes, de préférence avec attraction» ${ }^{24}$. Au contraire, les grands éditeurs, fidèles à la logique de sous-traitance, s'en remettent bien plutôt à leurs fournisseurs réguliers. Avec eux, ils peuvent être certains par exemple que sera respectée la liste non-écrite des sujets à éviter pour l'exportation

19. Henri de Brisay, «Mémoires de Fortuné Guignard, cinéiste», Hebdo-film, n 42-48, 19 octobre-14 décembre 1918. La somme de 25 francs se retrouve dans Francis Ambrière, "À l'aube du cinéma. Les souvenirs de Robert Péguy", l'Image, $\mathrm{n}^{\circ} 36,16$ septembre 1932.

20. Marc Mario, «Scénario et libretto», Ciné-Journal, n² 260, 16 août 1913.

21. Concernant la rémunération des jeunes auteurs dramatiques, voir Christophe Charle, Théâtres en capitales, Paris, Albin Michel, 2008, p. 161 et concernant les bonifications, voir Journal comptable Pathé, archives Fondation Jérôme Seydoux-Pathé, avril 1914: «Bonifications sur scènes. Rollini : 553 francs».

22. Fonds de la Commission de recherches historiques, Cinémathèque française, CRH-48.

23. Alain Carou, le Cinéma français et les écrivains. Histoire d'une rencontre, 1906-1914, Paris, École des Chartes / AFRHC, 2002, p. 212.

24. Ciné-Journal, n 238,15 mars 1913. 
dans les pays étrangers: pas d'adultère pour les pays anglo-saxons, pas de vol, pas de crime sanglant, pas de discussion père-fils pour l'Allemagne, pas d'attentat ou de complot pour la Russie ${ }^{25} \ldots$ Cependant, Grainville, dans le Courrier cinématographique déplore les conséquences du recours systématique aux mêmes hommes: «Les pauvres auteurs continuent à envoyer leurs œuvres [...], mais à part deux ou trois "fournisseurs" attitrés, les autres voient leurs envois retournés avec la mention: "Mille regrets" ${ }^{26}$. Le ressentiment redouble quand, un peu plus tard, le rédacteur du scénario croit reconnaître son idée plagiée dans une salle de cinéma. Le jeune écrivain René-Marie Hermant tente de fonder une société pour la protection des «librettistes cinématographiques» en arguant qu’ «il arrive souvent que des jeunes littérateurs ayant des idées neuves et ne voulant pas les transformer en romans, vont dans une des grandes maisons cinématographiques, et en toute confiance remettent leur libretto. Ces maisons, après lecture, déclarent que le livret est banal et qu'il ne peut passer, mais ayant pris note des idées et les modifiant quelquefois à peine, elles en tirent des films sensationnels ${ }^{27}$. Quelques années plus tard, Féval fils dénoncera, en tête des "pilleurs d'épaves scénariques», «le lecteur [celui qui chez l'éditeur est chargé de l'examen des scénarios] qui, associé à une horde de camoufleurs, repasse sous son propre nom et à son propre directeur les sélections adaptées et adoptées par lui ${ }^{28}$.

L'impossibilité pour un inconnu d'entrer en relation réelle avec un éditeur favorise les rentes de situation, la sous-traitance en cascade, et même quelques escroqueries. Des chargés d'affaires, qui de longue date se font fort de placer les textes littéraires ou dramatiques ${ }^{29}$, essaient de se diversifier dans le placement de scénarios cinématographiques. En 1910, Gance a placé ses deux premiers scénarios par un intermédiaire avant d'avoir un accès direct à plusieurs maison $\mathrm{s}^{30}$. Nora Jonuxi rapporte qu'elle «avai[t] un correspondant à Lyon [...]. Il m’avait dit “je suis en rapport avec Gaumont, Pathé, Éclair. Je vous placerai vos scénarios.” J'ai touché parfois cinquante francs. À Marseille, il y avait aussi quelqu'un qui s’occupait de placer le scénario " ${ }^{31}$. Mais la réputation de ce secteur est globalement déplorable et l'on n'accorde sans doute qu'une confiance limitée aux «agences Tricoche et Cacolet abonnées aux petites annonces: "On achète très cher les scénarios cinématographiques" "32. Un escroc est bien parvenu à soutirer à quelques naïfs des sommes substantielles, soi-disant pour placer leur scénario et le protéger au préalable contre les risques de plagiat en le publiant dans une revue ${ }^{33}$. Mais la seule initiative de quelque importance dans ce domaine revient à Edmond Bretel et René Le Somptier, deux

25. Jacques Roullet, «Les impressions d'un revenant», le Cinéma et l'Écho du cinéma réunis, Ge année, n 5, 2 février 1917.

26. Grainville, «Les abus», le Courrier cinématographique, $3^{\mathrm{e}}$ année, $\mathrm{n}^{\circ}$ 23, 14 juin 1913.

27. Courrier cité par Des Angles [Charles Le Fraper], «Les Librettistes cinématographiques », Comoedia, n 2025, 18 avril 1913.

28. Paul Féval, «Les adapt-adopteurs», le Courrier cinématographique, 9 année, n 27, 4 juillet 1919.

29. Les chargés d'affaires, ancêtres de la police privée, cumulent des activités diverses (renseignement commercial, enquêtes pour les familles, placement de titres mobiliers, placement de manuscrits...). Cf. Dominique Kalifa, Naissance de la police privée. Histoire des détectives privés (1843-1942), Paris, Plon 2000, rééd. Nouveau Monde, 2007.

30. Roger Icart, Abel Gance ou le Prométhée foudroyé, Lausanne, l'Âge d'homme, 1983, pp. 43-44.

31. Entretien avec Nora Jonuxi, op. cit.

32. Paul Féval, «Les adapt-adopteurs», op. cit. Tricoche et Cacolet est le titre d'un célèbre vaudeville dont les personnages sont deux agents d'affaires médiocres vivotant de petites escroqueries.

33. Eugène Meignen, «Une escroquerie», le Courrier cinématographique, $4^{\mathrm{e}}$ année, $\mathrm{n}^{\circ}$ 29, 25 juillet 1914. 
professionnels réputés qui créent en mai 1913 "Le Scénario français", société destinée à assurer le placement et la protection des scénarios ${ }^{34}$. Initiative un peu ambiguë en réalité: n'est-ce pas le moyen pour la société Cosmograph du même Bretel, encore peu connue, d'attirer à elle des propositions de scénarios de qualité et de s'en constituer un fonds à moindres frais?

Les plus grands bénéficiaires de la situation sont certainement les principaux fournisseurs de scénarios et, plus généralement, les hommes en place dans les grandes maisons. Les sous-traitants habituels qui ont un accès privilégié aux grands éditeurs de films peuvent profiter de l'existence à Paris d'un large vivier de jeunes auteurs à la recherche de travaux alimentaires pour sous-traiter à leur tour une partie de leur activité. Comme l'écrit un jeune auteur dramatique:

L'auteur peu connu est obligé de passer sous les fourches Caudines de [s fournisseurs attitrés], et savez-vous combien ces "trusteurs de la gaîté" offrent à l'obscur écrivain pour une bande de longueur moyenne? Eh bien, ils lui payent généreusement deux thunes, la journée d’un gâcheur de plâtre! [...] Ah! les combinaisons, les trafics! Voilà la plaie du théâtre, c'est aussi celle du cinéma. ${ }^{35}$

La tentation de recourir à des nègres existe sans doute aussi bien d'ailleurs chez les rédacteurs de scénarios salariés, tenus à des obligations aussi invraisemblables que «de produire six à dix pièces par semaine, et d'en lire cinquante autres $"$ ". Et sait-on si certains metteurs en scène qui passent dans les histoires du cinéma pour être les «auteurs» de leurs scénarios n’ont pas eu recours au même expédient? Si Gaumont fut condamné par la justice pour un plagiat assez visible d'une pièce de Paul Hervieu, c'est, selon Féval fils, parce que le metteur en scène Léonce Perret faisait écrire par un nègre les scénarios qu’il signait et que celui-ci se vengea un jour de lui en recopiant une œuvre protégée ${ }^{37}$. D'une autre manière, l'inégalité de traitement entre insiders et outsiders transparait aussi dans une anecdote relatée par Nora Jonuxi : bien qu’un rédacteur fût payé «au mois» pour écrire des scénarios pour la série Rigadin, véritable mine d'or pour la SCAGL, il était possible pour une rédactrice anonyme comme elle de vendre un scénario au tarif ordinaire de 50 ou 100 francs à condition de rester discrète ${ }^{38}$.

\section{Le pourcentage}

Entre le prix d'achat des scénarios et les bénéfices considérables engrangés par les films, un écart se creuse. Abel Gance note rageusement sur un scénario : «L'Électrocuté a été vendu au Film d'Art en 1911 pour 100 francs. Le Film d'Art vient de vendre le négatif à M. Wolff [Eclectic Films] $10000 »^{39}$. Au théâtre, la rémunération proportionnelle aux recettes est un principe intangible. Les premières revendications collectives émanent donc logiquement du Syndicat professionnel des auteurs dramatiques.

34. Annonce publiée dans le Courrier cinématographique, $3^{\mathrm{e}}$ année, ${ }^{\circ}$ 19, 17 mai 1913.

35. Lettre de Charles-Louis Janot, membre stagiaire de la Société des auteurs, citée dans "Cinématographes», Comodia, $\mathrm{n}^{\circ}$ 2368, 25 mars 1914.

36. Le Courrier cinématographique, 3e année, $\mathrm{n}^{\circ}$ 22, 7 juin 1913.

37. Paul Féval, «Les adapt-adopteurs», op. cit.

38. Entretien avec Nora Jonuxi, op. cit.

39. BnF, Arts du spectacle, Gance 32. 
Ce groupement s'est formé en 1907 afin de débattre des questions professionnelles plus démocratiquement qu’à la Société des auteurs (SACD). Celle-ci se compose en effet d'un groupe restreint de "sociétaires", auteurs à la situation bien établie qui seuls votent et choisissent les administrateurs en leur sein, et d'un nombre croissant de «stagiaires", auteurs débutants ou ayant eu une carrière difficile, soumis aux statuts de la Société mais sans possibilité d'influer sur ses choix ${ }^{40}$. Des stagiaires et même une partie des sociétaires reprochent à la Société des auteurs d'œuvrer surtout à renforcer l'accaparement des scènes par les "gros" au détriment des "petits " ${ }^{41}$. En décembre 1911, le syndicat envoie à tous ses membres un rapport d'André Heuzé sur la perception d'un pourcentage de la recette des salles de cinéma au bénéfice des auteurs ${ }^{42}$. En fait, dès 1909, un éphémère "Syndicat des auteurs cinématographistes français" a formulé cette proposition ${ }^{43}$, tout simplement calquée sur les droits d'auteur au théâtre. Mais le modèle

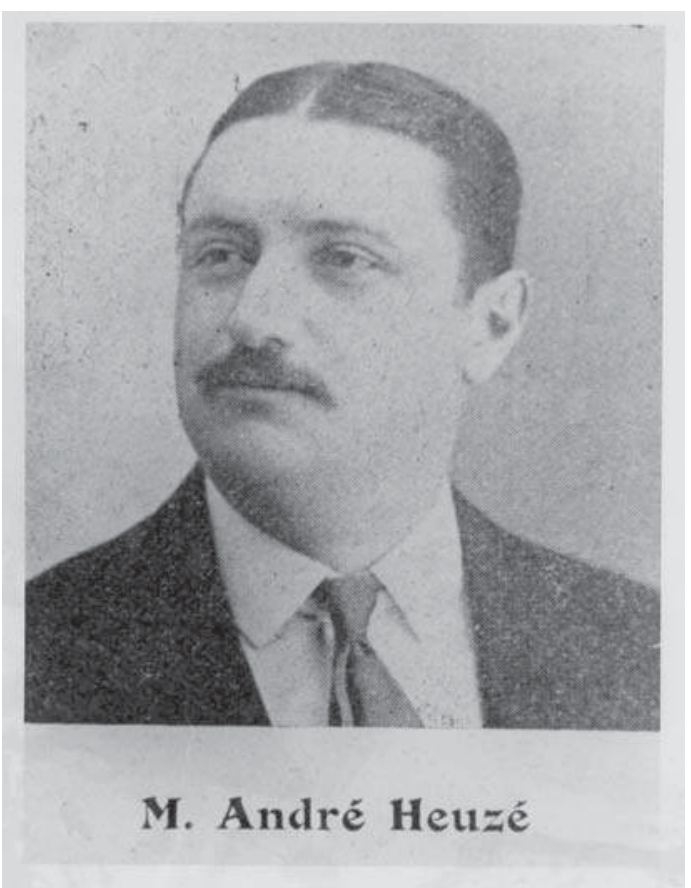

Portrait d'André Heuzé, Collection Lacassin, s.d. de rémunération du «scénario d'auteur" adopté par Le Film d'Art et la SCAGL - au métrage et au nombre de copies - pouvait paraitre alors plus pratique et déjà fort attirant. Si moins de trois ans plus tard Heuzé défend à nouveau le pourcentage - comme on ne tardera pas à l'appeler, - c'est, justifie-t-il, parce que la location des films est désormais si bien organisée que de moins en moins de copies suffisent à réaliser de plus en plus de projections, et donc de plus en plus de recettes ${ }^{44}$. Le syndicat mène si bien campagne que la SACD s'empare du sujet pour désamorcer cette concurrence et qu'elle entreprend rapidement d'étendre ses statuts au cinéma, autrement

40. De 1887 à 1907, le nombre des sociétaires passe de 393 à 302, tandis que celui des stagiaires passe de 800 à 4058 ! L'accès au sociétariat se trouve presque bloqué à partir de 1904, quand est instaurée la cooptation. Lire à ce propos Jean Bayet, la Société des auteurs et compositeurs dramatiques, thèse de doctorat, Paris, 1908, pp. 221-222.

41. Cf. par exemple Adolphe Le Pailleur, la Question théâtrale par un auteur stagiaire, Paris, à compte d'auteur, 1907.

42. Rapport d'André Heuzé sur la perception des droits, envoyé avec l'annuaire 1912 du syndicat professionnel des auteurs dramatiques, cité dans le Courrier cinématographique, $2^{\mathrm{e}}$ année, $\mathrm{n}^{\mathrm{o}} 1,1^{\mathrm{er}}$ janvier 1912.

43. Annonce publiée dans Kinema, $\mathrm{n}^{\circ}$ 12, 17 mai 1909.

44. On retrouvera plus tard le même argument dans la bouche de Pierre Decourcelle: «les éditeurs français qui louent actuellement les films de leur production aux exploitants moyennant des prix uniformes et qui varient seulement d'après leur ancienneté de représentation, paraissent décidés à renoncer à ce système pour lequel ils sont obligés de se baser sur les petits établissements et qui ne leur donne pas de produits suffisants, par suite de l'augmentation de leurs frais; et à y substituer la location moyennant une part proportionnelle des recettes" (procès-verbal de la séance de la commission de la SACD du 13 novembre 1916, archives SACD). 
dit d'obliger tous ses membres à passer par son intermédiaire pour gérer leurs affaires cinématographiques, afin d'instaurer à terme la perception en salles pour tous ${ }^{45}$.

Les «pourcentistes» vont subir une telle volée de bois vert dans la presse corporative que l'on pourrait croire que toute l'industrie du cinéma est unie contre cette revendication. La situation est un peu plus complexe. Les exploitants sont assurément hostiles au pourcentage, synonyme pour eux de nouvelle taxe et de droit de regard d'une société de perception sur leurs livres de comptes. Comme ils forment sans doute le gros des abonnés aux journaux corporatifs, ceux-ci ne peuvent pas ouvertement se désolidariser de leurs intérêts. Cela n'empêche pas de noter des nuances entre le Courrier cinématographique de Charles Le Fraper, résolument «anti-pourcentiste», et Ciné-Journal de Georges Dureau qui prône le dialogue. Les rédacteurs en chef des principaux titres (les deux précités et le Cinéma et l'Écho du cinéma réunis de Georges Lordier) étant eux-mêmes membres stagiaires de la Société des auteurs, ils comprennent les revendications de leurs confrères, à défaut de les partager. Le dilemme est sans doute plus vif encore chez les employés des éditeurs de films qui appartiennent par ailleurs à la SACD comme membres stagiaires, et qui peuvent redouter de se trouver obligés de choisir entre la profession à laquelle ils aspirent et celle dont ils vivent ${ }^{46}$. Les éditeurs de films, quant à eux, ont des intérêts et des options nettement divergents. Gaumont est et restera obstinément opposé à la reconnaissance d'un statut au collaborateur littéraire pour les films muets - alors qu'il y est tout ouvert pour les films sonores. Mais Charles Jourjon, directeur d'Éclair, part très tôt à l'offensive pour tenter de briser l'accaparement des grandes signatures par Pathé ( via Le Film d'Art et la SCAGL), accaparement perçu comme un nouvel effort de la firme au coq pour empêcher l'émergence de concurrents sérieux. À cet effet, il propose dès 1909 aux autres éditeurs de s'accorder sur des droits d'auteur calculés sur une base plus avantageuse, combinant un pourcentage des ventes et un pourcentage des recettes d'exploitation. Ce mode de rémunération uniforme pour tous les éditeurs mettrait à bas les monopoles de la SCAGL et du Film d'Art, puisque ces sociétés, proposant des conditions moins intéressantes, verraient leurs auteurs se retourner contre elles. Ensuite, au fil des années, et surtout à mesure qu'ils sont plus dépendants du marché intérieur français, certains éditeurs affichent de plus en plus clairement leur intérêt à ce que se mette en place une perception sur les recettes des salles, à condition qu'elle leur profite également. En effet, le tarif unique de location des copies apparaît de plus en plus inapproprié à mesure que l'exploitation cinématographique se diversifie, car il est calculé d'après les capacités des petits exploitants. Une piste pour rentabiliser les grands films de qualité serait donc de faire payer aux grandes salles un supplément proportionnel à leurs recettes ${ }^{47}$. Des éditeurs, des loueurs et des fournisseurs de scénarios se réunissent ainsi en 1914 pour discuter d'un mode de perception profitable à tous ${ }^{48}$.

45. Ce point est largement développé dans le Cinéma français et les écrivains..., op. cit., pp. 265-300.

46. Dufilm, "Du scénario», Ciné-Journal, n 299, 16 mai 1914. Cette crainte est, chez Gaumont, celle de Louis Feuillade et de Jacques Roullet (Louis Feuillade, retour aux sources, éd. Alain Carou et Laurent Le Forestier, Paris, AFRHC, 2007, pp. 53-54; procès-verbal de l'assemblée des stagiaires de la SACD du $1^{\text {er }}$ décembre 1913, archives SACD).

47. Cf. par exemple Guy de Téramond dans la Patrie, art. cité dans Ciné-Journal, nº 300, 23 mai 1914.

48. "Cinématographes", Comoedia, $\mathrm{n}^{\circ}$ 2351, 10 mars 1914. Les fournisseurs de scénarios en question sont ceux du Syndicat des auteurs et compositeurs cinématographiques, conduits par Marc Mario (voir plus loin). 
Cela permet d'y voir un peu plus clair dans la stratégie d'André Heuzé. Car à lire la presse corporative, on pourrait se demander pourquoi un rédacteur de scénarios bien implanté dès 1904 aurait pris le parti de se couper de l'industrie en prenant la tête du combat pour le pourcentage. En fait, en 1910, Heuzé s'est associé à trois des principaux animateurs du Syndicat professionnel pour fonder Le Film des Auteurs, qui pourrait être une tentative de créer une troisième société spécialisée dans le "scénario d'auteur» en prenant appui sur le réseau constitué par le syndicat, principalement des auteurs de vaudevilles (peu représentés à la SCAGL). Heuzé a donc déjà rompu avec Pathé à ce moment et joue probablement de relations avec d'autres éditeurs. Le Film des Auteurs rencontrant un succès des plus limités, le combat pour le pourcentage peut contribuer à lui redonner une position d'intermédiaire entre le milieu des auteurs dramatiques et une partie des éditeurs de films. Il n’a pas forcément escompté que la Société des auteurs l'en déposséderait rapidement. Néanmoins il n'a pas abandonné tout espoir: après avoir fondé en 1914 la revue le Film, en 1916, devant les lenteurs de la SACD, il lance une nouvelle société dont l'objet serait rien moins que de placer les scénarios auprès des éditeurs, d'assurer la perception sur la recette des exploitants et de reverser les recettes aux auteurs ${ }^{49}$.

\section{L'affirmation d'un groupe professionnel}

En décrétant le pourcentage, les administrateurs de la Société des auteurs ne prévoyaient peutêtre pas qu'ils se heurteraient à l'opposition de confrères romanciers en passe de devenir les nouveaux maîtres de la sous-traitance des scénarios. Leur décision apparait en effet surtout comme une bonne manière faite aux directeurs de théâtre en cherchant à peser sur les finances des salles de cinéma, car ils n'ont aucune relation avec l'industrie cinématographique. Plusieurs romanciers populaires parmi les plus lus viennent au contraire de s'y lancer comme dans une nouvelle branche d'activité à côté du feuilleton, de la librairie et de la scène ${ }^{50}$. Marc Mario et Léon Sazie - ce romancier brusquement lancé par le succès de Zigomar en 1911 - enchainent les éditoriaux dans le Cinéma de Georges Lordier. Ils honnissent le pourcentage qui les priverait de la liberté de négocier avec les éditeurs de films, favoriserait les fournisseurs de scénarios non-membres des sociétés d'auteurs, et au final risquerait d'obliger les professionnels de la littérature à travailler clandestinement pour le cinéma. À la suite de cette vive polémique, le premier groupement de défense des intérêts des professionnels du scénario voit le jour en juin 1913. On retrouve au sein de ce «Syndicat des auteurs et compositeurs cinématographiques» des romanciers populaires comme Guy de Téramond et Paul Féval fils, et plusieurs anciens animateurs du Film des Auteurs et du Syndicat professionnel des auteurs dramatiques - qui vient de s'auto-dissoudre sous pression de la SACD ${ }^{51}$.

49. Procès-verbal de la séance de la commission de la SACD du 17 novembre 1916, archives SACD.

50. Cf. le rapport de Jules Mary à la Société des gens de lettres: «Le cinéma vous offre un des moyens d'étendre votre action sur un domaine encore en friche." (cité dans Ciné-Journal, n² 298, 16 mai 1914).

51. "Cinématographes», Comoedia, n² 2088, 20 juin 1913. 


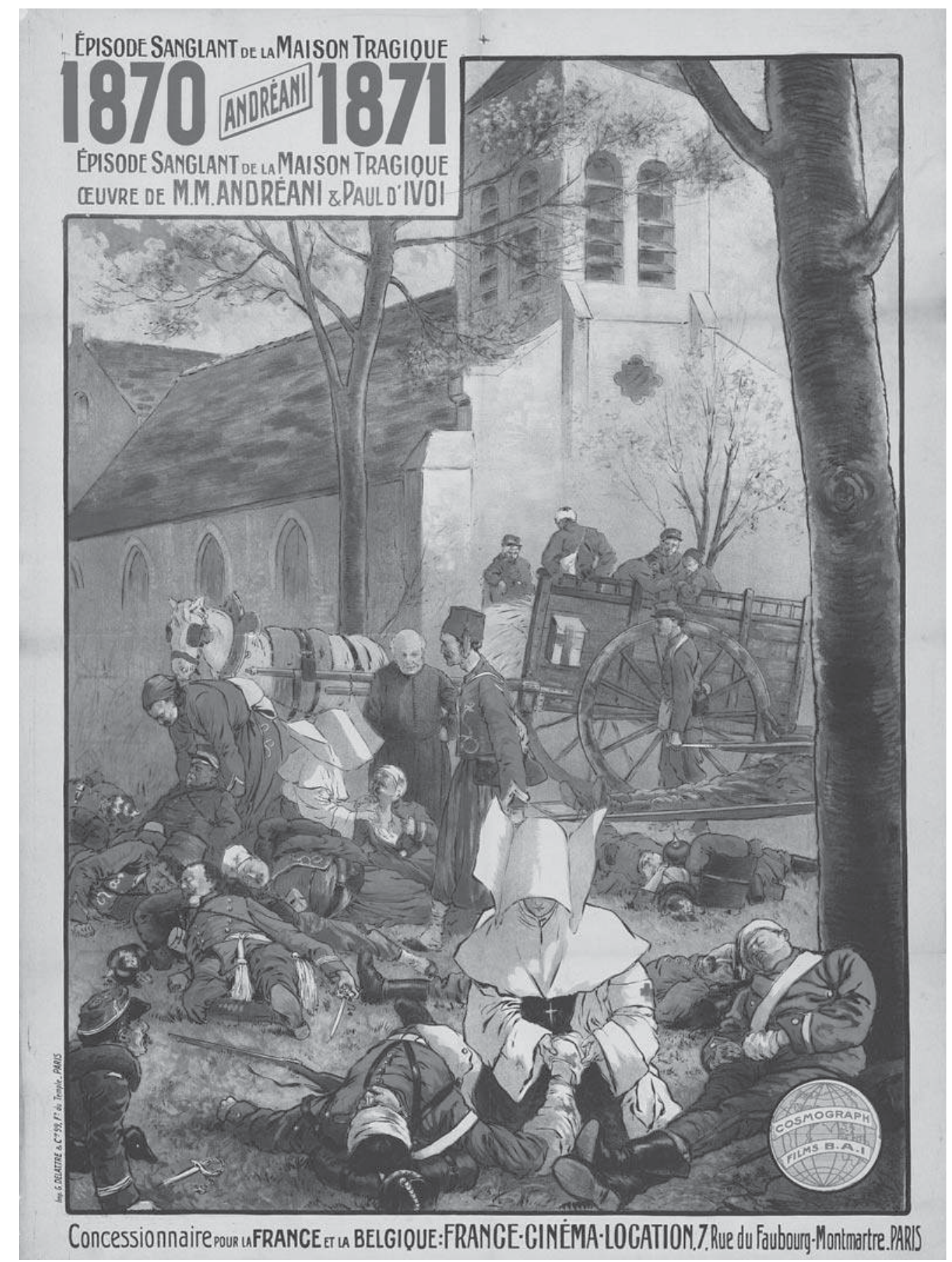

Affiche du film 1870-1871 d'Henri Andréani, 1913. Coll. Cinémathèque française. 
Cette affirmation est la face visible de l'émergence d'un noyau de professionnels, que courtisent les nouveaux éditeurs des années 1912-1914, spécialisés dans le grand film dramatique: «Les Grands Films populaires» de Lordier, Tallandier, Cosmograph, Aubert... Ces hommes se connaissent déjà. Certains travaillent en collaboration, d'autres se concurrencent sans merci dans l'industrie du roman. Anecdote révélatrice: on pourrait croire que si le thème des Dernières cartouches inspire deux films sortis au printemps 1914 (les Dernières cartouches et 1870-1871), c'est parce que la Revanche est dans l'air du temps. En réalité, Jules Mary porte plainte devant la Société des auteurs à l'encontre de Paul d'Ivoi, parce que celui-ci, au cours d'une discussion à la Société des gens de lettres, a eu vent de son projet de film avec Tallandier et s'est empressé d'aller souffler l'idée (titre compris!) à Cosmograph $^{52}$.

Cela est révélateur de nouvelles attentes de la part des éditeurs. Car d'Ivoi n'a cru pouvoir aller aussi loin que parce que le sujet des Dernières cartouches fait partie du fonds commun de l'Histoire de France. Aussi connus que soient ces romanciers, leur rôle n'est pas d'apporter une idée originale signée - ce à quoi se résumait souvent le «scénario d'auteur» - mais une histoire précisément architecturée en fonction des besoins du cinéma. Le travail d'adaptation d'un roman préexistant peut ainsi être confié désormais à l'un d'eux. Aubert a par exemple promis pas moins de mille francs à Mario pour l'adaptation de la Curée de Zola ${ }^{53}$. Des émoluments aussi inhabituels ne rémunèrent pas une signature - celle de Zola suffit - mais un savoir-faire technique. Technicité dont Decourcelle et Gugenheim reconnaissent la rareté, dans une lettre à Jules Mary: «Tu es un des rares auteurs qui savent faire un scénario cinématographique, et une adaptation faite par toi est un sûr garant de réussite ${ }^{54}$.

Une cause majeure du processus de professionnalisation est indéniablement l'allongement des métrages, qui requiert des scénarios plus élaborés. Les écrivains suffisamment motivés pour les écrire commencent à se distinguer clairement de ceux - notamment des dramaturges ${ }^{55}$ - dont la contribution se réduira à fournir des idées originales. Marc Mario le souligne à l'envi: «Tout auteur, ayant le don d'imagination qui l'a fait ce qu'il est, peut enfanter un scénario; pour être du bâtiment, il faut connaître à fond la partie, il faut être initié complètement au Cinéma pour écrire un libretto définitif " $"$. Et de définir le professionnel du scénario avant tout comme un adaptateur, capable d'exploiter une «simple idée» tout aussi bien qu'une œuvre dramatique et littéraire pour les «tritur[er] selon les nécessités cinématographiques»: son métier consiste à savoir tirer des idées des autres des plans de films exécutables.

52. Procès-verbal de la séance de la commission de la SACD du 9 avril 1914, archives SACD. Finalement, d'Ivoi, obligé de reconnaitre ses torts, change le titre.

53. Procès-verbal de la séance de la commission de la SACD du 30 janvier 1914, archives SACD.

54. Lettre du 9 juin 1916, reproduite dans Bulletin des Amis du roman populaire, ${ }^{\circ}{ }^{15}, 1991$, p. 8.

55. Sur l'attitude des dramaturges, cf. Alain Carou, le Cinéma français et les écrivains..., op. cit., pp. 262-232.

56. Marc Mario, «Scénario et libretto", op. cit. Le terme de "scénariste» n’a pas encore été forgé à cette époque, on parle donc plutôt de «librettiste». Mario n’est donc peut-être pas le premier à parler de «libretto » en matière de cinéma, mais il l'est sans doute à l'employer par différence (et non par équivalence) avec «scénario ". 


\section{Méthodes et concours : former de nouveaux professionnels}

Au même moment, la presse cinématographique commence à traiter du scénario comme d'une technique à acquérir. L'auteur le plus intéressant à ce sujet est Léon Demachy.

C'est lui qui, sous l'anagramme d'Yhcam, publie en 1912 l'étude «La cinématographie au point de vue théâtral ». Il y affirme nettement que «l'auteur dramatique, avant d'aborder le livret de cinéma, doit avant tout faire un nouvel apprentissage». Dans ce texte, connu pour contenir l'idée novatrice que le spectateur du film muet fait une expérience active en mettant des mots sur les images qui défilent devant lui, la notion d'une présence silencieuse du verbe dans le film se retrouve, d'une autre manière, dans les quelques lignes concernant le travail du scénario : «Le livret fourni par l'auteur [...] doit être absolument complet; il doit comporter, non seulement les détails de mise en scène voulus par l'auteur, mais encore le dialogue, car le geste ou le jeu de physionomie de l'acteur cinématographiste ne peut être vrai que s'il accompagne le mot juste [...] " ${ }^{57}$. L'importance de la qualité littéraire du scénario pour assurer la qualité du jeu des acteurs apparaît également primordiale à G. Pierre-Martin, qui voudrait que le scénario "passionne l'artiste» "par l'exacte description des lieux, par la logique des situations, par la solidité du dialogue mimé», et même que des parties en soient versifiées afin de «mieux envelopper l'âme des répétitions dans l'ambiance musicale du verbe initiateur du mouvement général de l'œuvre ${ }^{58}$.

Ces considérations précoces cèdent rapidement le pas à une approche plus technique, au service du metteur en scène, où priment la concision, la lisibilité du récit et la nature visuelle des scènes. Comme l'écrit le mystérieux Dufilm, «un scénario n'a pas besoin d'être littéraire. Il faut seulement indiquer la marche à suivre ${ }^{59}$. "Technique du scénario", une série d'articles publiée au début de 1914 par Demachy, se présente comme une véritable méthode, adaptée de Technique of the Photoplay de l'Américain Epes W. Sargent ${ }^{60}$. Les règles d'écriture qu'il définit concernent d'abord les sujets à éviter, soit parce qu'ils seraient rédhibitoires pour les ventes à l'étranger: questions politiques, religieuses ou sociales, crime ou acte de violence à l'écran; soit parce que les éditeurs de films ne les confient qu’à des spécialistes: sujets impliquant d'employer des animaux savants, pièces à trucs, exploitation "à chaud" des faits d'actualité, adaptations d'œuvres littéraires. Elles portent ensuite sur la manière d'organiser les tableaux: l'histoire doit être traitée dans l'ordre sans retour en arrière dans le temps, les personnages doivent être présentés et leur caractère bien défini dès les premiers tableaux, et il convient d'estimer le métrage de chaque tableau pour ne pas excéder la valeur d'une ou deux bobines. Elles concernent, enfin,

57. Yhcam [Léon Demachy], «La cinématographie au point de vue théâtral», Ciné-Journal, n 192, 27 avril 1912.

58. G. Pierre-Martin, «L'Art cinématographique (Études comparatives)", le Cinéma et l'Écho du cinéma réunis, ${ }^{\circ}$ 26, 26 juillet 1912.

59. Dufilm, «Du scénario», Ciné-Journal, nº 298, 9 mai 1914.

60. Léon Demachy, "Technique du scénario", le Courrier cinématographique, $4^{\mathrm{e}}$ année, n 2-8, 10 janvier-21 février 1914. En juillet 1911, Sargent a inauguré dans le Moving Picture World la rubrique "Technique of the Photoplay», dont les articles sont ensuite compilés en un ouvrage, plusieurs fois réédité; cf. Torey Liepa, «Figures of Silent Speech: Silent Film Dialogue and the American Vernacular, 1909-1916», PhD, New York University, 2008, p. 124. Demachy ne cache pas sa dette à l'égard de Sargent et signe parfois du pseudonyme d'Americus. 
les normes de présentation écrite: argument, distribution des rôles, schéma des tableaux, scénario proprement dit. Chez Demachy comme dans d'autres articles contemporains, la "visualisation ${ }^{61}$ mentale du film est présentée comme la clé d'un scénario réussi : «Le véritable scénariste voit ses personnages en action, il les évoque pour ainsi dire pendant qu'ils vivent et il comprend ce qu'ils font, ce qu'ils disent sans avoir besoin de les entendre parler. [...] Quand il écrira son scénario, il se servira de sa mémoire visuelle en décrivant simplement "ce qu'il a vu" "62.

Les concours de scénarios pourraient servir à la diffusion de ces codes, à travers les règlements et les conseils donnés aux candidats. L'article de Demachy prend d'ailleurs plusieurs fois en exemple un concours organisé par la Cines italienne et très richement doté. Mais si en Italie les concours ont le soutien des éditeurs, qui visent à remédier à une carence de scénarios et à exercer une forme de mécénat culturel $^{63}$, en France les - rares - initiatives reviennent à la presse corporative seule. Or à quoi bon avoir un scénario primé s'il n’a pas d'éditeur? Le concours organisé par le Cinéma-Théâtre tire les conséquences de ce paradoxe en proposant au gagnant de produire le film aux frais du journal et de lui remettre le négatif ${ }^{64} \ldots$ L'opération a manifestement une visée publicitaire. Néanmoins le règlement confirme que les enjeux sous-jacents à ce type de concours sont tout autres qu'en 1909, quand l'Argus phonocinéma lançait un appel à idées afin que le public pût concourir à remédier à la "crise du sujet" dont il était alors abondamment question ${ }^{65}$. En 1914, l'acquisition d'une discipline d'écriture et la distinction des travaux les plus aboutis sont à l'ordre du jour.

Pourquoi la presse corporative cherche-t-elle à élargir le recrutement des rédacteurs de scénarios si les éditeurs de films n'en sont guère demandeurs ${ }^{66}$ ? Probablement s'agit-il d'abord de répondre à une demande, voire à des récriminations, des lecteurs - et notamment des exploitants qui se piquent d'apporter leur écot à l'industrie du film ${ }^{67}$. Demachy ouvre ainsi son article sur le portrait d'un quidam qui jette son premier scénario sur le papier comme il lui vient et se vexe de rester sans nouvelles de l'éditeur auquel il l'a envoyé. On peut se demander s'il ne s'agit pas également pour le

61. Le terme, inusité alors, est un anglicisme que Demachy revendique comme un emprunt de plus aux méthodes développées outre-Atlantique.

62. Émile Patient, "Le scénario au point de vue composition ", le Cinéma et l'Écho du cinéma réunis, n 223, 15 sept. 1916.

63. Le concours de la Cines s'ancre ainsi dans les mêmes logiques que les «séries d'art» italiennes, produites par des aristocrates pour diffuser les valeurs de haute culture dans le vaste public. Le jury composé de hautes personnalités françaises et italiennes - par exemple le directeur de la Villa Medicis - veut encourager des œuvres «susceptible [s] d'éveiller dans la foule un frisson de vraie beauté» (entretien avec Albert Besnard paru dans l'Intransigeant, cité dans le Cinéma et l'Écho du cinéma réunis, $\mathrm{n}^{\circ}$ 82, 19 septembre 1913). Le premier prix (25000 francs, une véritable fortune) sera partagé entre deux sujets antiques: le Trésor de Rhampsinite, d'Americano Scarlatti, et Persée, de Maurice Magre. Sur le scénario en Italie, cf. Silvio Alovisio, Voci del silenzio: la sceneggiatura nel cinema muto italiano, Turin, Museo nazionale del cinema/ Il Castoro, 2005.

64. Cinéma-Théatre, nº 3, 7 février 1914.

65. "Un concours de scénarios", Argus phono cinéma, n 101, février 1909.

66. À l'exception peut-être du concours organisé en 1912 par le Cinéma, puisque son directeur (Lordier) est également éditeur de films.

67. Par exemple, Gabriel Kaiser envoie aux journaux un scénario pour publication afin que les éditeurs «n’ignorent pas que ce scénario est ma propriété d'auteur et sa reproduction interdite dans tous les pays sans mon autorisation" (le Courrier cinématographique, $4^{\mathrm{e}}$ année, $\mathrm{n}^{\circ} 18,9$ mai 1914). 


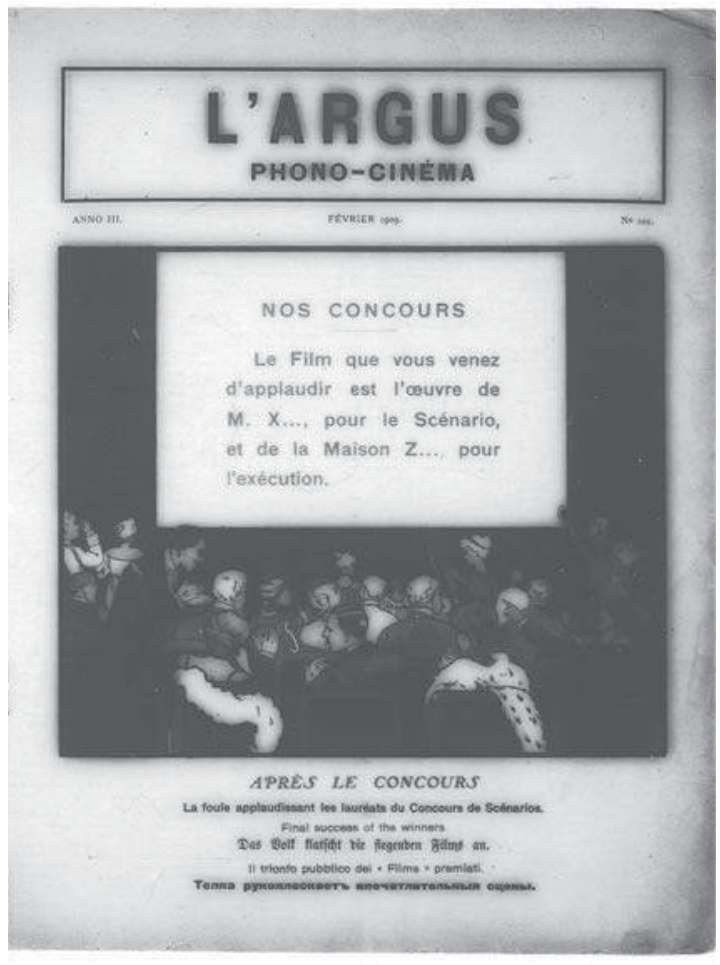

Couverture de l'Argus Phono-cinéma, n 101, février 1909.
Courrier cinématographique d'allumer d'intelligents contrefeux aux revendications de la Société des auteurs. Charles Le Fraper, son directeur, signe plusieurs éditoriaux où il affirme qu'avoir un passé littéraire ne prépare en rien à écrire pour le cinéma. Publier la «méthode Demachy» revient à affirmer que n'importe qui s'intéresse au cinéma pourrait devenir un bon rédacteur de scénarios à condition de faire l'effort de s'en approprier la technique.

\section{Quand le scénario fait l'auteur du film}

La guerre plonge le cinéma français dans une crise grave et prolongée, alors même qu'il commence à apparaître comme un instrument d'influence stratégique. Dans la presse, la faiblesse des scénarios revient très souvent comme l'une des racines les plus profondes du mal, davantage par exemple que la vétusté des théâtres de prise de vues. Sont incriminés corrélativement le manque de professionnels, car le monde littéraire a eu tendance à croire qu'il s’agissait de vendre un simple canevas, et la faiblesse des tarifs pratiqués par les éditeurs. "Messieurs les auteurs, je vous en prie, apprenez à écrire directement pour le cinéma", s'exclame Armand Bour, nouveau venu à la mise en scène ${ }^{68}$. Contrepartie normale, écrit Meignen, "un scénario intéressant, vraiment cinématographique et intelligemment soigné, se paierait aussi cher que le droit d'adapter une pièce ou un roman ${ }^{69}$. A cet égard, la différence de rémunération entre la France et les États-Unis ou l'Italie est abondamment soulignée. L'est aussi le manque de discernement des lecteurs employés par les maisons d'édition ${ }^{70}$. L'auto-critique de la profession se traduit par exemple par l'organisation d'un «concours des scénarios refusés» par le Cinéma en juin 1919.

Cette situation contribue à convertir de nouveaux éditeurs au prélèvement d'un pourcentage sur les recettes d'exploitation partagé entre eux et les scénaristes ${ }^{71}$. Le quasi-monopole acquis par la

68. Armand Bour, «Pour la suprématie du scénario », le Film, n 98, 28 janvier 1918.

69. Edmond Meignen, «Les scénaristes», le Cinéma et l'Écho du cinéma réunis, nº 218, 11 août 1916.

70. Cf. par exemple Abel Rubi, «De bons scénarios SVP», Ciné-Journal, n 344, 18 mars 1916.

71. L'autre raison, primordiale, est la plus grande dépendance des éditeurs français par rapport aux recettes faites sur le marché intérieur. 
SCAGL ne fait plus obstacle, mis en cause qu'il est de la part des écrivains privés de leur liberté de négociation avec d'autres éditeurs, et de la part de Pathé qui ne voit plus d'intérêt à son partenariat avec Decourcelle $^{72}$. Dans ses interventions sur l'avenir du cinéma français, Charles Pathé préconise que les "auteurs de scénarios» soient assez bien payés pour pouvoir vivre de l'écriture de deux ou trois scénarios par $\mathrm{an}^{73}$. Le groupement d'intérêts entre éditeurs et professionnels du scénario fait de grands progrès à ce moment ${ }^{74}$.

Au-delà de l'aspect strictement économique des "droits d'auteur», c'est la question des «droits de l'auteur» - selon une distinction proposée par Marc Mario - qui se trouve posée avec une vigueur inédite. Des progrès dans la qualité des scénarios semblent en effet conditionnés au respect de la propriété des rédacteurs de scénarios et à la publication systématique de la signature, bref à «tout ce qui peut contribuer à étendre leur réputation, à concourir à la diffusion de leur nom $»^{75}$. Déjà inclus dans les statuts de la Société des auteurs cinématographistes de 1913 et invoqués avant guerre, ces objectifs sont indissociables du processus de professionnalisation ${ }^{76}$. D'élément de réclame, mis en avant par choix de l'éditeur (c'était le principe du «scénario d'auteur»), la signature devient un enjeu pour les rédacteurs de scénarios désireux de renforcer leur renom. Jules Mary réclame ainsi que la SACD l'impose «au bas des œuvres cinématographiques» et sur les affiches et programmes ${ }^{77}$.

Inversement, la demande de retrait de signature se trouve invoquée quand le scénariste juge que les modifications apportées au scénario par le metteur en scène ou le producteur dénaturent son travail et nuiraient à sa réputation ${ }^{78}$.

Comment s'assurer un contrôle sur l'«exécution » de l'idée visuelle complète que vise désormais à être le scénario? Jean Joseph-Renaud conseille d'établir un découpage très précis, «où tout est indiqué, où rien n'est laissé dans le vague, [et qui] constitue le film lui-même tel qu'il sera une fois réalisé

72. Le contrat entre Pathé et la SCAGL prévoyait notamment un minimum de recettes garanti pour cette dernière. Pathé rompt par étapes entre 1916 et 1918. La SCAGL devient alors une société secondaire jusqu’à sa disparition en 1924. 73. Réponse à l'enquête publiée dans la Renaissance politique, littéraire et artistique, 14 octobre 1916, n ${ }^{\circ} 1$, pp. 19-20; propositions reprises et développées dans son Étude sur l'évolution de l'industrie cinématographique, Paris, Établissements Pathé Frères, 1918.

74. Procès-verbal de la séance de la commission de la SACD, 29 novembre 1916, archives SACD. Cf. aussi Georges Lordier, «La société des éditeurs et auteurs cinématographistes», le Cinéma et l'Écho du cinéma réunis, n 231, 10 novembre 1916. Le 4 octobre 1918, Bernède annonce à la SACD qu'un accord a été trouvé entre loueurs et éditeurs (procèsverbal de la séance de la commission, archives SACD).

75. Marc Mario, «Le nom de l'auteur», Ciné-Journal, nº 347, 8 avril 1916.

76. R. Serrano, "Plus d'œuvres anonymes au cinéma! Un danger», le Cinéma et l'Écho du cinéma réunis, $\mathrm{n}^{\circ} 187,7$ janvier 1916. La revendication avait été formulée avant-guerre, cf. R. Laubier, op. cit.; Charles Le Fraper, «Rendons à César...", le Courrier cinématographique, 3e année, nº 19, 17 mai 1913; Georges Dureau, "Le droit de signer appartientil aux auteurs de scénarios?», Ciné-Journal, n 233, 1 ${ }^{\text {er }}$ février 1913.

77. Procès-verbal de la séance de la commission de la SACD, 8 décembre 1916, archives SACD. Cf. aussi Paul Féval, «Exigez la signature», le Cinéma et l'Écho du cinéma réunis, n 200, 7 avril 1916

78. Alain Carou, «Usage(s) de la notion d'auteur par les gens de cinéma, de l’institutionnalisation aux années 1940 ", dans Actes du colloque "Lauteur du film, histoire et archéologie d'une notion» (Christophe Gauthier et Dimitri Vezyroglou, dir. ), Paris, AFRHC, à paraître en 2012. 
et monté». Ce "découpage technique qui est le style d'un scénario et d'un film» engagera le metteur en scène et le producteur ${ }^{79}$.

Mais Joseph-Renaud et d'autres sont tentés par une autre voie, celle de devenir leurs propres metteurs en scène ${ }^{80}$. Comme Tristan Bernard l'affirme de la façon la plus nette:

pour être un auteur du ciné, il faut s'astreindre à mettre en scène son scénario, ou tout au moins en surveiller étroitement la mise en scène, en donnant ses indications au photographe, spécialiste technique véritable celui-là [c'est-à-dire: à la différence du metteur en scène, “fausse” spécialité professionnelle] [...] Le découpage des films, l'interprétation, le choix du décor, la mise en scène en un mot, c'est le travail de l'auteur, c'est l'écriture du film. ${ }^{81}$

Le passage de rédacteurs de scénarios à la mise en scène était chose courante avant la guerre ${ }^{82}$, mais l'idée nouvelle d' «auteur de film» complet consiste à affirmer l'unité de principe des deux fonctions, contre la logique de spécialisation ${ }^{83}$. Constituée à l'automne 1917, la Société des auteurs de films indique l'émergence d'un groupe de professionnels économiquement indépendants ou en recherche d'indépendance, dont l'activité est tournée de fait pour les uns davantage vers la mise en scène, pour les autres davantage vers l'écriture de scénarios, mais ne se limite pas par principe à l'une ou l'autre ${ }^{84}$. Amorcée en 1912-1914, la constitution des scénaristes en groupe distinct n'est plus d'actualité.

Le processus de normalisation de l'écriture s'en est-il trouvé affecté lui aussi? La question mériterait plus ample examen, mais c'est loin d'être évident. Par exemple, le fait qu’un scénario soit aisément lisible par d'autres que celui qui l'a écrit conserve force de loi. En 1921, Léon Moussinac proclame certes qu'un véritable scénario est un brouillon intime et n'a rien à voir avec une nouvelle forme de littérature: «indéchiffrable pour quiconque, son auteur seul peut s'y reconnaître ${ }^{85}$. Ce faisant, il

79. Jean Joseph-Renaud, «Auteurs et directeurs», le Courrier cinématographique, $9^{\mathrm{e}}$ année, $\mathrm{n}^{\circ} 8,1^{\mathrm{er}}$ mars 1919. L'auteur s'attire des réponses défendant le droit du metteur en scène et du producteur, qui l'amènent à se dédire partiellement dans le Courrier cinématographique, $9^{\mathrm{e}}$ année, $\mathrm{n}^{\circ}$ 12, 29 mars 1919.

80. Cf. par exemple, sur L’Herbier, Alain Carou, "À la conquête de la souveraineté. L'idée d'auteur selon Marcel L'Herbier ", dans Laurent Véray (dir. ), Marcel L'Herbier, l'art du cinéma, Paris, AFRHC, 2008, pp. 135-150.

81. Tristan Bernard, discours à l'assemblée générale de la SACD, 18 mai 1917, reproduit dans Annuaire de la Société des auteurs et compositeurs dramatiques, 1916-1917, p. 489.

82. Citons les exemples de Jacques Roullet, Daniel Riche, Michel Carré, Gaston Ravel.

83. Celle-ci n’a pas disparu du tout, mais elle s'ancre dans des projets de réindustrialisation du cinéma national encore vacillants, notamment les Cinéromans. Là, les scénarios sont écrits par des romanciers populaires qui restent à l'écart de la mise en scène (ce sont plutôt les activités d'écriture qu'ils concentrent, dans la mesure où ils assurent la novélisation de leurs scénarios).

84. Cf. Xavier Loyant, «la Société des auteurs de films, 1917-1929», thèse de l'École des Chartes, 2009 (résumé consultable en ligne: http://theses.enc.sorbonne.fr/2009/loyant).

85. Léon Moussinac, "Cinématographie», Mercure de France, $n^{\circ} 561,1^{\text {er }}$ novembre 1921, p. 786. À ce moment, Moussinac est directeur littéraire des éditions de la Lampe Merveilleuse, qui tentent d'inventer une forme d'équivalent littéraire des films qui ne soit ni la publication de scénarios ni une novélisation conventionnelle à la manière des « romanscinéma". 
n'ignore pas que depuis 1918 environ les «cinégraphistes» comme Gance, Delluc, L'Herbier publient volontiers leurs scénarios dans les revues afin qu'ils puissent servir de modèle. Le lecteur destinataire n'est plus seulement ou plus du tout un producteur ou un metteur en scène, mais le public ou les confrères. Moussinac lui-même, en 1920, a qualifié de "quasi parfait» le scénario de la Fête espagnole de Louis Delluc parce qu'il se lit comme un «conte de sang, de vol et de mort» doué d'un «rythme» propre ${ }^{86}$. Un rédacteur de Ciné-Journal résume bien cette nouvelle manière d'envisager les scénarios. Il voit dans leur circulation le moyen d'aider la production à "s'épurer" par l'exemple. Il ajoute que cela permettrait d'éviter que «de très belles œuvres, après une fugitive apparition sur l'écran, disparaissent à jamais dans le plus noir oubli " ${ }^{87}$. Les scénarios n’ont pas perdu en "valeur d'échange», ils en auraient plutôt acquis une voire deux supplémentaires: celle de pouvoir servir d'exemple et celle de constituer un répertoire de l'art cinématographique.

86. Léon Moussinac, "Cinématographie», Mercure de France, n 526, 15 mai 1920, p. 250.

87. Louis Franco-Allain, «La survivance du scénario», Ciné-Journal, n 527, 18 septembre 1919. Demachy avait été précurseur en 1912: «Pourquoi les très bons films, ceux qui possèdent une valeur intrinsèque réelle, ne viendraient-ils pas former un Répertoire, répertoire qui resterait et pourrait être repris? Pourquoi les livrets hors ligne ne seraient-ils pas imprimés, avec leur mise en scène, tout comme les livrets de théâtre, et ne viendraient-ils pas constituer la Bibliothèque du Théâtre cinématographique?» (Yhcam [Léon Demachy], «La cinématographie considérée au point de vue théâtral [IV : Des livrets ou scénarios. Conditions générales]», Ciné-Journal, nº 193, 1er mai 1912). 